\title{
Eribulin-based treatment in patients affected by sarcomas: a case series
}

\author{
Giovanni Grignani*,1 \& Lorenzo D'Ambrosio' \\ ${ }^{1}$ Candiolo Cancer Institute, FPO - IRCCS, Strada Provinciale, 142 km 3,95, Candiolo, 10060 TO, Italy \\ *Author for correspondence: giovanni.grignani@ircc.it
}

First draft submitted: 25 September 2019; Accepted for publication: 18 October 2019; Published online: 16 December 2019

Keywords: doxorubicin • eribulin mesylate • gemcitabine • ifosfamide • leiomyosarcoma • liposarcoma

Sarcomas are a rare and heterogeneous group of tumors of mesenchymal origin arising from soft tissues (STS) or, less frequently, from the bone [1]. Liposarcoma (LPS) accounts for up to $18 \%$ of all STS. LPS can occur in almost any part of the body, but more than half of the cases involve the thigh and up to a third involve the abdominal cavity. Dedifferentiated LPS, along with well-differentiated LPS/atypical lipomatous tumors, represent the largest group of LPS. Computed tomography and MRI represent the gold standards in diagnosis, staging and preoperative evaluation of LPS, while the only potentially curative approach to LPS is the complete surgical resection of the tumor with negative microscopic margins. Unfortunately, the tendency for relapse in LPS is high and the disease often progresses to tumors that are not surgically amenable due to their extension or anatomical relationships to neighboring structures, which means the outcomes in terms of patient survival remain poor.

\section{Existing treatment}

Systemic treatments for locally advanced or metastatic STS are limited and often associated with significant toxicity [2]. Doxorubicin is still the reference treatment in first-line chemotherapy. The addition of ifosfamide to doxorubicin is related to a better response rate and progression-free survival (PFS), but not to an improvement in terms of overall survival (OS) [3]. Gemcitabine plus docetaxel has been evaluated in comparison with doxorubicin, showing no superiority in terms of survival and a significant toxicity [4]. Dacarbazine has some activity in advanced STS, in particular leiomyosarcoma (LMS) and solitary fibrous tumors [2]. More recently, eribulin and trabectedin, two compounds derived from the sponge Halichondria okadai and from the tunicate Ecteinascidia turbinata, respectively, were studied in advanced sarcomas. In second or subsequent lines of therapy, these marine-derived drugs became available based on the results of randomized Phase III trials, which used dacarbazine or placebo as a comparator.

Treatment indications differ among STS histological subtypes: pazopanib has been approved for all STS other than LPS, while trabectedin has been approved for both LPS and LMS in USA and for all STS from second-line therapies in Europe [5,6]. However, these drugs are associated with limited benefits in terms of objective response and survival.

Eribulin mesylate (eribulin) is a synthetic analog of halichondrin B, a nontaxane microtubule-destabilizing anticancer agent [7]. Eribulin has been approved in USA and in Europe for the treatment of metastatic breast cancer since 2010. In a subsequent randomized, open label, multicentric Phase III trial (E7389-G000-309), which compared eribulin with dacarbazine in 452 patients affected by advanced LPS or LMS, and who had already received at least two lines of therapy, eribulin significantly improved OS but not PFS. However, a prespecified subgroup analysis showed a substantial benefit in terms of both OS and PFS for patients with the LPS histotype [8,9]. The trial also revealed a very manageable toxicity profile in the eribulin arm versus the comparator, dacarbazine. Hence, in 2016, both the US FDA and the EMA approved eribulin for the treatment of patients with advanced LPS that had previously received an anthracycline-based treatment. 


\section{Reported case studies}

The case reports included in this supplement provide an update on the use of eribulin in clinical practice for patients affected by LMS or LPS localized in different anatomical regions. Some special clinical scenarios are discussed to promote the correct use of the drug in challenging patients, including those submitted to nephrectomy.

In more detail, Aliberti $e t$ al. reported the case of a 51-year-old woman affected by advanced uterine LMS (one of the most common histotypes of uterine sarcoma) with liver, bone and lung metastases. After a first-line doxorubicin-based treatment, a second-line treatment with trabectedin and a third-line treatment with gemcitabine, the patient was finally treated with eribulin. After 6 cycles, a CT scan showed partial remission of the liver lesion and stability of other lesions, with no severe side effects and a good quality of life.

In the report authored by Martorana et al., the authors described the treatment of a patient with a dedifferentiated LPS, initially misdiagnosed as a high-grade LMS, which progressed after several lines of therapy before eribulin treatment. In addition to routine histopathological analysis, disease re-classification as dedifferentiated LPS was also confirmed through DNA and RNA sequencing. The disease remained stable after completing eight cycles of eribulin as a fifth-line treatment that was substantially well tolerated by the patient, confirming the favorable safety profile even in heavily pretreated patients.

Tober $e t$ al. reported their clinical experience of two patients affected by retroperitoneal LPS, both submitted to concomitant right nephrectomy, who experienced a long-lasting control of the disease through an eribulin-based therapeutic regimen. Authors observed minor side effects, including neutropenia (during the first cycles of therapy) and constipation.

Sbrana et al. described another case of advanced, retroperitoneal dedifferentiated LPS. In more detail, treatment with eribulin followed a first-line therapy with anthracycline and continued for about 1 year with no major adverse events, with the exclusion of an episode of grade- 2 anemia at the beginning of the fourth cycle.

Paioli et al. described six clinical cases of patients with advanced LPS that received eribulin as third- or fourth-line therapy. Eribulin treatment was well tolerated, with no grade 3-4 toxicity or therapy delay. Notably, the authors used a full dose of eribulin in three elderly patients ( $\geq 65$ year old), reporting no additional adverse event.

Finally, Miano et al. reported the case of a heavily pretreated male patient affected by left funiculus LPS and submitted to three surgical interventions, radiotherapy on the lesion of the penile bulb for satellite nodules and an epirubicin + ifosfamide chemotherapy treatment for six cycles. Eribulin was administered for nine cycles and a significant reduction of the lesions was achieved after four cycles of therapy, with a good profile of tolerability.

All of these reports highlighted eribulin treatment effectiveness with a relatively low cost in terms of toxicity in pretreated patients affected by different subtypes of sarcomas, including LPS and uterine LMS. The reports also highlighted that the intervention of a dedicated pathologist, along with the use of molecular analyses, could lead to a better classification of these tumors and, possibly, to a personalized treatment approach. Finally, the optimal sequence of available therapies is far from having been identified in the treatment of advanced sarcomas. In this context, some of the authors raised the hypothesis that an earlier use of eribulin, given its relatively low toxicity profile and efficacy, should at least be considered in a more personalized strategy.

\section{Author contributions}

Both authors were involved in the writing and revision of this manuscript and have provided final approval to submit.

Financial \& competing interests disclosure

The authors have no relevant affiliations or financial involvement with any organization or entity with a financial interest in or financial conflict with the subject matter or materials discussed in the manuscript. This includes employment, consultancies, honoraria, stock ownership or options, expert testimony, grants or patents received or pending, or royalties.

Medical writing support was provided by Amalia Forte, PhD, on behalf of Content Ed Net, and funded by Eisai.

\section{References}

1. Fletcher CDM, Bridge JA, Hogendoorn P, Mertens F. WHO Classification of Tumours of Soft Tissue and Bone (4th Edition). IARC Press, Lyon, France (2013).

2. Casali PG, Abecassis N, Aro HT et al. Soft tissue and visceral sarcomas: ESMO-EURACAN Clinical Practice Guidelines for diagnosis, treatment and follow-up. Ann. Oncol. 29(Suppl. 4), iv268-iv269 (2018).

3. Judson I, Verweij J, Gelderblom H et al. Doxorubicin alone versus intensified doxorubicin plus ifosfamide for first-line treatment of advanced or metastatic soft-tissue sarcoma: a randomised controlled Phase III trial. Lancet Oncol. 15(4), 415-423 (2014). 
4. Seddon BM, Whalen J, Strauss SJ et al. Gemcitabine and docetaxel versus doxorubicin as first-line treatment in previously untreated advanced unresectable or metastatic soft-tissue sarcomas (GeDDiS): a randomised controlled Phase III trial. Lancet Oncol. 18(10), 1397-1410 (2017).

5. Demetri GD, von Mehren M, Jones RL et al. Efficacy and safety of trabectedin or dacarbazine for metastatic liposarcoma or leiomyosarcoma after failure of conventional chemotherapy: results of a Phase III randomized multicenter clinical trial. J. Clin. Oncol. 34(8), 786-793 (2016).

6. van der Graaf WT, Blay JY, Chawla SP et al. Pazopanib for metastatic soft-tissue sarcoma (PALETTE): a randomised, double blind, placebo controlled Phase III trial. Lancet 379(9829), 1879-1886 (2012).

7. Bai RL, Paull KD, Herald CL, Malspeis L, Pettit GR, Hamel E. Halichondrin B and homohalichondrin B, marine natural products binding in the vinca domain of tubulin. Discovery of tubulin-based mechanism of action by analysis of differential cytotoxicity data. $J$. Biol. Chem. 266(24), 15882-15889 (1991).

8. Schoffski P, Chawla S, Maki RG et al. Eribulin versus dacarbazine in previously treated patients with advanced liposarcoma or leiomyosarcoma: a randomised, open-label, multicentre, Phase III trial. Lancet 387(10028), 1629-1637 (2016).

9. Demetri GD, Schöffski P, Grignani G et al. Activity of eribulin in patients with advanced liposarcoma demonstrated in a subgroup analysis from a randomized Phase III study of eribulin versus dacarbazine. J. Clin. Oncol. 35(30), 3433-3439 (2017). 